\title{
Vascularised human skin equivalents as a novel in vitro model of skin fibrosis and platform for testing of antifibrotic drugs
}

\author{
Alexandru-Emil Matei $(1),{ }^{1}$ Chih-Wei Chen, ${ }^{1}$ Lisa Kiesewetter, ${ }^{2}$ \\ Andrea-Hermina Györfi, ${ }^{1}$ Yi-Nan Li, ${ }^{1}$ Thuong Trinh-Minh, ${ }^{1}$ Xiaohan Xu, ${ }^{1}$ \\ Cuong Tran Manh, ${ }_{1}^{1}$ Toin van Kuppevelt, ${ }^{3}$ Jan Hansmann, ${ }^{2,4}$ Astrid Jüngel, ${ }^{5}$ \\ Georg Schett (1) , ${ }^{1}$ Florian Groeber-Becker, ${ }^{2,6}$ Jörg H W Distler
}

Handling editor Josef $\mathrm{S}$ Smolen

- Additional material is published online only. To view please visit the journal online (http://dx.doi.org/10.1136/ annrheumdis-2019-216108)

For numbered affiliations see end of article.

Correspondence to Dr Jörg H W Distler, Department of Internal Medicine 3 -

Rheumatology and Immunology, Friedrich-Alexander-University Erlangen-Nürnberg (FAU) and University Hospital Erlangen, Erlangen D-91054, Germany; joerg.distler@uk-erlangen.de

A-EM, C-WC, FG-B and JHWD contributed equally.

Received 30 July 2019 Revised 12 September 2019 Accepted 13 September 2019 Published Online First 20 September 2019

\section{ABSTRACT}

Objectives Fibrosis is a complex pathophysiological process involving interplay between multiple cell types. Experimental modelling of fibrosis is essential for the understanding of its pathogenesis and for testing of putative antifibrotic drugs. However, most current models employ either phylogenetically distant species or rely on human cells cultured in an artificial environment. Here we evaluated the potential of vascularised in vitro human skin equivalents as a novel model of skin fibrosis and a platform for the evaluation of antifibrotic drugs.

Methods Skin equivalents were assembled on a three-dimensional extracellular matrix by sequential seeding of endothelial cells, fibroblasts and keratinocytes. Fibrotic transformation on exposure to transforming growth factor- $\beta$ (TGF $\beta$ ) and response to treatment with nintedanib as an established antifibrotic agent were evaluated by quantitative polymerase chain reaction (qPCR), capillary Western immunoassay, immunostaining and histology.

Results Skin equivalents perfused at a physiological pressure formed a mature, polarised epidermis, a stratified dermis and a functional vessel system. Exposure of these models to TGF $\beta$ recapitulated key features of SSc skin with activation of TGF $\beta$ pathways, fibroblast to myofibroblast transition, increased release of collagen and excessive deposition of extracellular matrix. Treatment with the antifibrotic agent nintedanib ameliorated this fibrotic transformation.

Conclusion Our data provide evidence that vascularised skin equivalents can replicate key features of fibrotic skin and may serve as a platform for evaluation of antifibrotic drugs in a pathophysiologically relevant human setting.

\section{INTRODUCTION}

Fibrotic disorders such as systemic sclerosis (SSc) are complex multifactorial diseases that develop as interplay of different cell types with each other and with their local microenvironment. ${ }^{1}$ Preclinical model systems are essential to improve our understanding of the underlying pathophysiology of fibrotic diseases and to test novel therapeutic approaches. The most frequent approaches are simulating fibrotic conditions either in vitro with human cell culture systems or in vivo with mouse models. Most of the current cell culture systems

\section{Key messages}

What is already known about this subject?

- Many current model systems of skin fibrosis are of limited predictive value as they rely either on phylogenetically distant species or oversimplified culture conditions that deprive the cells of a three-dimensional matrix and of interactions with other cell types.

- These limitations provide major challenges to preclinical development and may contribute to the significant rate of negative clinical trials in systemic sclerosis (SSc).

\section{What does this study add?}

- We describe vascularised skin equivalents as an advanced model of human skin with a fully polarised epidermal layer, a stratified dermis and a functional vascular system with physiological perfusion pressures.

- Human skin equivalents can be induced to undergo fibrotic transformation and resemble key features of SSc skin with accumulation of extracellular matrix, fibroblast to myofibroblast transition and aberrant activation of transforming growth factor- $\beta$ (TGF $\beta$ ) signalling.

- Vascularised skin equivalents can predict response to antifibrotic therapies as shown by inhibition of fibroblast activation and of extracellular matrix deposition in response to nintedanib.

How might this impact on clinical practice or future developments?

- Vascularised skin equivalents may serve as a novel model to better mimic the complex interplay of different cells in fibrotic skin and may also provide a platform for the evaluation of antifibrotic drugs in a pathophysiologically relevant human setting with improved predictive value.

are two dimensional (2D), where cells are cultured on stiff, flat plastic surfaces. ${ }^{2}$ Although studies in $2 \mathrm{D}$ cell culture systems have provided important insights into cellular responses of individual cell types to defined stimuli, they have several 
important limitations: they cannot mimic (patho)physiologically relevant cell-matrix interactions, interactions between different cell types, presence of nutrient gradients and signalling pathways related to these processes. ${ }^{3-5}$ Moreover, culture of adherent cell types on plastic surfaces exposes them to an unphysiologically stiff environment, which alters central cellular responses such as response to cytokines, organisation of the cytoskeleton, migration and proliferation. ${ }^{67}$ Cellular responses in $2 \mathrm{D}$ culture models may thus not always reliably predict the pathological processes in fibrotic tissues. Mouse models overcome several of these limitations, such as lack of cell-cell and cell-matrix interactions, absence of gradients and excessive tissue stiffness, but contain other potential pitfalls. There are many differences between murine and human immune systems-dendritic epidermal $\mathrm{T}$ cells, for example, are only found in mouse. ${ }^{89}$ Moreover, not all pathways are conserved between mice and men and individual molecules may exert different functions in those signalling cascades. ${ }^{10}$ Pathophysiological studies and preclinical test programmes for novel antifibrotic approaches may thus benefit from novel in vitro test systems with primary human cells to overcome some of the limitations of conventional 2D cell culture systems and mouse models.

Here, we report an in vitro test system for skin fibrosis, generated by adapting a previously described vascularised skin equivalent. ${ }^{11}$ This model employs primary human fibroblasts, keratinocytes and endothelial cells that resemble all functional skin layers affected by fibrosis in a three-dimensional (3D) environment and provides a functional vascular system with physiological perfusion. We demonstrate that this engineered human skin equivalent can be converted into fibrotic skin by exposure to transforming growth factor- $\beta$ (TGF $\beta)$. The induction of fibrosis is prevented by nintedanib, demonstrating that this model is a suitable test system for candidate antifibrotic drugs. This vascularised in vitro human skin equivalent may thus be an interesting addition to the arsenal of preclinical models of skin fibrosis.

\section{MATERIALS AND METHODS}

Materials and methods are described in the online supplementary information file.

\section{RESULTS}

Vascularised human skin equivalents reproduce main characteristics of human skin relevant for the pathogenesis of skin fibrosis

We generated vascularised human skin equivalent models and evaluated their suitability as a test system for generation and prevention of skin fibrosis by exposing them to TGF $\beta$ and/or nintedanib (figure $1 \mathrm{~A}$ ). ${ }^{11}$

For this, we refined a previously described bioreactor design to ensure higher reproducibility and throughput. We modified the bioreactor body from a round to a rectangular shape, while adapting its inner dimensions to allow easy access to the cannulas of the artery and vein of the skin equivalent during culture (figure 1B,C,E). Thepolydimethylsiloxane (PDMS) lid of the

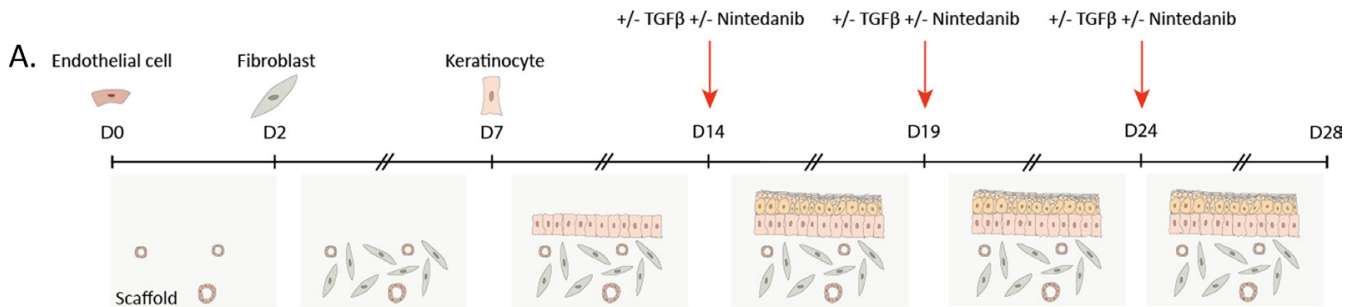

B.

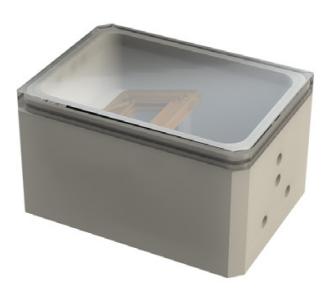

E.

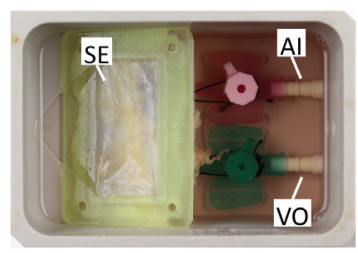

C.

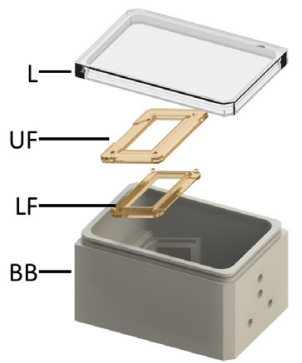

F.

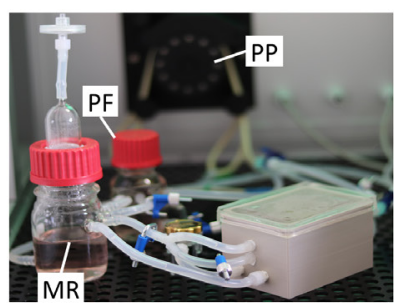

D.

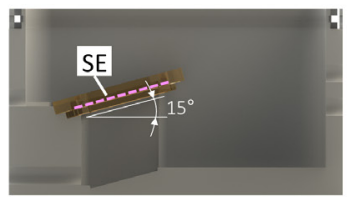

G.

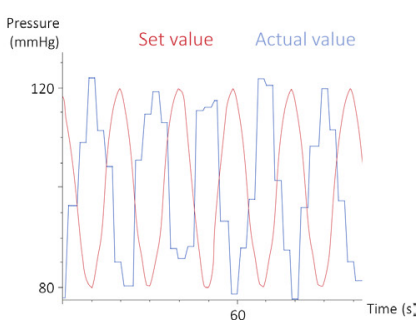

Figure 1 Assembly of the vascularised human skin equivalent and characterisation of the optimised bioreactor system. (A) Schematic overview of the generation of the human skin equivalent and its conversion to fibrotic skin. (B-D): Schematic representation of the optimised bioreactor and its contents $(B, C)$, with the matrix at a $15^{\circ}$ angle (D). (E) Picture illustrating the bioreactor contents: the human skin equivalent with cannulated supplying artery (red cannula) and vein (green cannula) connected to the perfusion system. (F) Picture illustrating the perfusion system and the bioreactor containing the human skin equivalent. (G) Pressure curves measured at the arterial end of the human skin equivalent (blue) following the set values (red) and resembling the physiological human systemic blood pressure. Al,arterial BB,bioreactorbody; L, lid; LF, lowerframe; MR, medium reservoir; PF, pressurebalance flask; PP, peristaltic pump; SE, skin equivalent; TGF $\beta$, transforming growth factor- $\beta$; UF, upper frame; VO, venous outlet. 

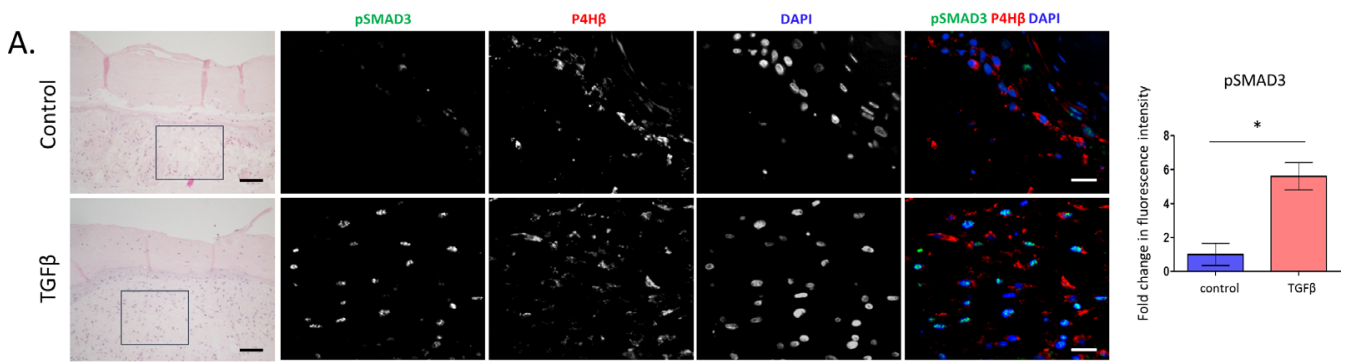

B.

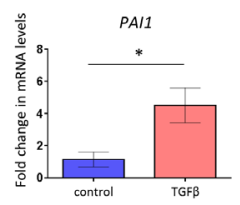

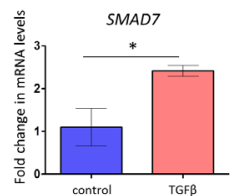

C.

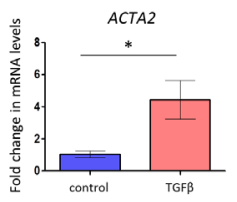

D.

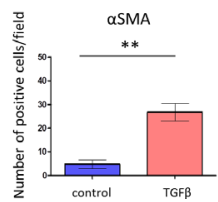

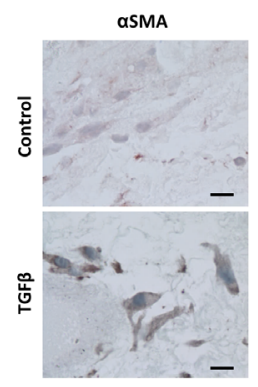

Figure 2 Systematic evaluation of TGF $\beta$ signalling in vascularised human skin equivalent models. (A) Activation of Smad signalling as shown by immunofluorescence staining for pSMAD3 (green) and costaining with prolyl-4-hydroxylase- $\beta$ (red) and DAPI (blue), at 600-fold magnification (scale bars $=25 \mu \mathrm{m}$ ). Quantification of pSMAD3 signal intensity and HE stainings (200-fold magnification, scale bars $=100 \mu \mathrm{m})$ is included. $(\mathrm{B}) \mathrm{mRNA}$ levels of the prototypical TGF $\beta / S M A D$ target genes PAI-1 and Smad7. (C) mRNA levels of ACTA2 (which encodes for $\alpha$ SMA). (D) Quantification of myofibroblast counts and representative $\alpha$-SMA stainings counterstained with haematoxylin at 1000 -fold magnification (scale bars=20 $\mu \mathrm{m}$ ). $\alpha$-SMA, $\alpha$-smooth muscle actin; DAPI, 4',6-diamidino-2-phenylindole; TGF $\beta$, transforming growth factor- $\beta$.

bioreactor provided a tight sealing while allowing direct macroscopic inspection of the skin model and its vessels during culture, which permits rapid detection of technical problems (figure 1C). Furthermore, we introduced an angled orientation of the skin equivalent to prevent entrapment of air bubbles underneath the matrix that would lead to uneven medium supply (figure 1D).

Perfusion at the arterial end was ensured by connecting the bioreactor system containing the skin models to a fluidic circuit; a peristaltic pump and a pressure sensor ensured pressure generation and control that resemble the physiological systemic blood pressure (figure 1F,G).

After 1 month of in vitro culture, the microscopic structure of the vascularised skin equivalent resembled that of human skin (online supplementary figure 1A). The keratinocytes had fully differentiated to form a polarised human epidermis with physiological location of cytokeratin-14 in the basal layer and cytokeratin-10 in the suprabasal layers and presence of a corneous layer (online supplementary figure 1B). Laminin-5 staining demonstrated that the epidermis is separated from the underlying dermis by a continuous basal membrane in our model as it is in human skin (online supplementary figure 1B). Fibroblasts migrated in the scaffold and distributed uniformly to form a dermal equivalent, while the superficial fibroblasts remain in close proximity to the epidermis.

Endothelial cells lined the pre-existent vessel structures, as shown by the positive staining for CD31 in the dermis (online supplementary figure 1C). Fibroblasts identified as vimentin-positive or $\mathrm{P} 4 \mathrm{H} \beta$-positive cells migrated in close proximity to the endothelial cells, facilitating interactions (online supplementary figure 1C). The vessel density in skin equivalent models, although lower than in human skin from healthy donors, was similar to the density in skin from patients with SSc (online supplementary figure 2). Podoplanin-positive lymphatic endothelial cells formed lymphatic vessels in vascularised skin equivalents that persisted until the end of the experiment (online supplementary figure 3 ).

\section{Exposure to TGF $\beta$ induces fibroblast to myofibroblast transition and deposition of extracellular matrix (ECM) in vascularised human skin equivalents}

Aberrant TGF $\beta$ signalling is both sufficient and required to induce fibrosis in various organs including skin. ${ }^{12}$ We thus evaluated whether exposure to pathophysiologically relevant concentrations of TGF $\beta$ would convert normal skin equivalents to fibrotic skin equivalents.

We first aimed to confirm that incubation with TGF $\beta$ activates the transcription of common downstream targets of TGF $\beta$. Indeed, we observed increased phosphorylation and nuclear translocation of Smad3 in P4H $\beta$-positive cells in the dermis of vascularised human skin equivalents (figure $2 \mathrm{~A}$ ). Moreover, the TGF- $\beta$ target genes PAI1 and SMAD7 were upregulated in tissue homogenates from fibrotic skin equivalents as compared with the control (figure 2B).

We further analysed fibroblast to myofibroblast transition in the vascularised skin models exposed to TGF $\beta$. The mRNA levels of ACTA2 and the number of $\alpha$-smooth muscle actin ( $\alpha$-SMA)positive cells were significantly higher in the TGF $\beta$-stimulated skin equivalents than in controls (figure 2C,D), thus reproducing the activated state of fibroblasts observed in fibrotic skin. ${ }^{13}$

We next investigated the deposition of ECM components in TGF $\beta$-exposed human skin equivalents. The mRNA levels of COL1A1, COL1A2 and fibronectin were significantly increased 
A.
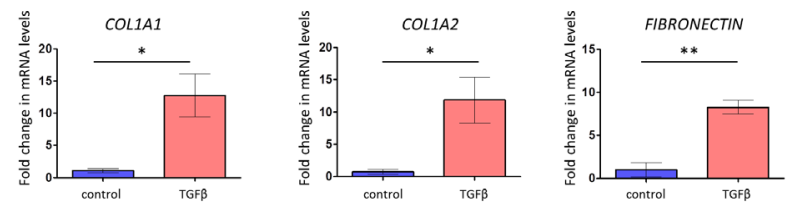

B.
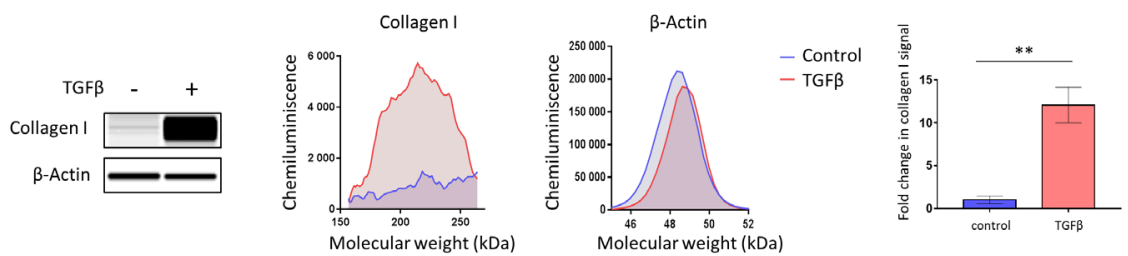

C.

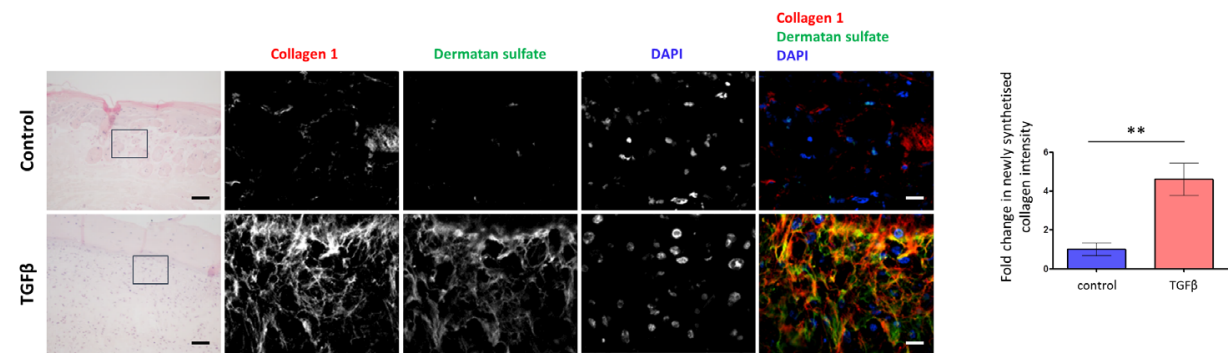

Figure 3 Fibrotic remodelling of the vascularised human skin equivalent in response to TGF $\beta$. (A) Upregulation of the mRNA levels of COL1A1, COL1A2 and fibronectin as major ECM proteins in the skin. (B) Representative western blot for type I collagen, with $\beta$-actin as housekeeping gene. Chemiluminescence versus molecular weight plots and quantification are included. (C) Immunofluorescence stainings of dermatan sulfate to identify human type I collagen in the vascularised human skin equivalent, at 1000 -fold magnification (scale bars $=20 \mu$ m), counterstained with DAPI. HE stainings (200-fold magnification, scale bars $=100 \mu \mathrm{m}$ ) and quantification of collagen type I signal colocalising with dermatan sulfate are included. DAPI, 4',6-diamidino-2-phenylindole; ECM, extracellular matrix; TGF $\beta$, transforming growth factor- $\beta$.

in the TGF $\beta$-stimulated skin equivalents (figure 3A). Moreover, the levels of type 1 collagen protein, evaluated by capillary Western immunoassay, were highly upregulated in the TGF $\beta$-exposed human skin equivalent (figure $3 \mathrm{~B}$ ).

However, all antibodies directed against collagen type 1 that we tested could not reliably discriminate between human (newly synthesised) and porcine (pre-existent) collagen, since collagen type 1 protein is evolutionarily highly conserved (data not shown). To identify the newly synthesised collagen type 1 and determine its relative contribution to the collagen levels in the two experimental conditions, we used an indirect approach. Dermatan sulfate was previously shown to be an excellent surrogate readout for de novo deposition of type 1 collagen, due to its association with newly formed collagen fibrils and its absence from the matrices. ${ }^{14}$ We confirmed the specificity of this approach in our setting by showing with immunofluorescence staining that collagen type 1 of porcine origin could be detected in the acellular matrix by western blot, while the dermatan sulfate signal was absent (online supplementary figure 4). The dermatan sulfate and collagen type I double positive signal highlighted the organisation of newly synthesised collagen as prominent fibres in the TGF $\beta$ group, whereas in the control group double positive signals were minimal. The intensity of collagen type I signal colocalising with dermatan sulfate was much stronger in the TGF $\beta$ group than in control, proving the increased de novo synthesis and deposition of collagen in TGF $\beta$-exposed skin equivalents (figure 3C).

\section{Nintedanib ameliorates fibrosis in the vascularised human skin equivalent}

To evaluate its potential use as a platform to test candidate antifibrotic drugs, we analysed whether TGF $\beta$-induced fibrosis could be reduced by treatment with nintedanib, a multiple tyrosine kinases inhibitor with proven antifibrotic effects. ${ }^{15-17}$

We first evaluated whether nintedanib can inhibit TGF $\beta$ downstream signalling and TGF $\beta$-induced fibroblast to myofibroblast transition. In the dermis of TGF $\beta$-exposed, nintedanib-treated skin equivalents, phosphorylation and nuclear translocation of Smad3 in P4H $\beta$-positive cells was decreased, and the TGF- $\beta$ target genes PAI1 and SMAD7 were downregulated as compared with TGF $\beta$-stimulated and untreated skin models (figure 4A,B). Nintedanib treatment also inhibited the TGF $\beta$-induced upregulation of ACTA2 mRNA levels and led to lower numbers of $\alpha$-SMA-positive cells (figure 4C,D).

We subsequently analysed the deposition of ECM components in TGF $\beta$-exposed, nintedanib-treated skin models in comparison with stimulated and vehicle-treated equivalents. TGF $\beta$-induced upregulation of COL1A1, COL1A2 and fibronectin mRNA levels and total collagen type I protein were significantly decreased by nintedanib treatment (figure 5A,B). In the TGF $\beta$-stimulated, nintedanib-treated group, the signal intensity of newly synthesised collagen was reduced in comparison to the TGF $\beta$-stimulated, vehicle-treated group (figure 5C).

\section{DISCUSSION}

Vascularised human skin equivalents resemble key features of human skin. The keratinocytes in the epidermal layer are polarised, and dermis and epidermis are separated by a basal membrane. Fibroblasts are located as single cells in the dermal layer, embedded into a physiological matrix. Endothelial cells form a functional, perfused vascular network comprising both blood and lymphatic vessels. This model thus includes all major resident cell types of human skin in typical topographic order 

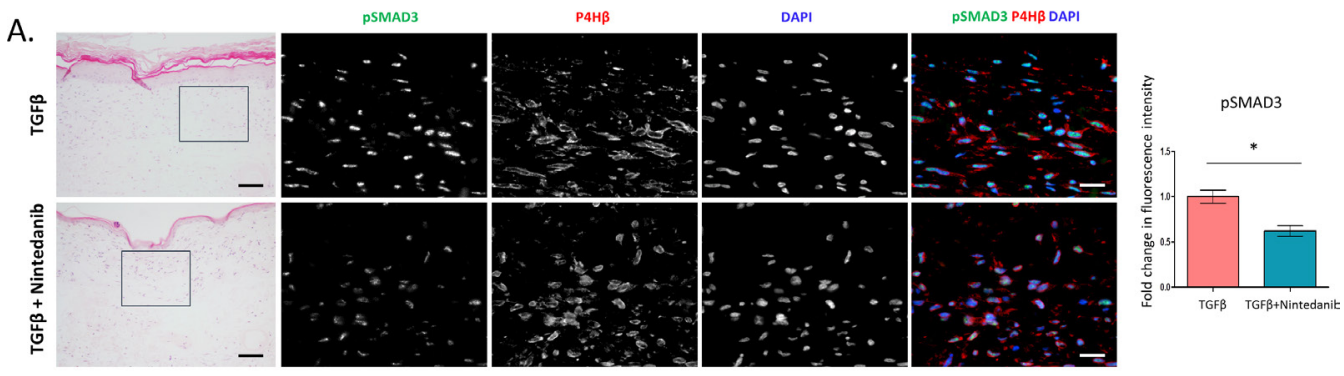

B.
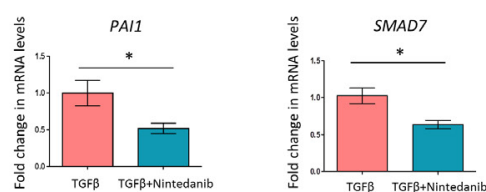
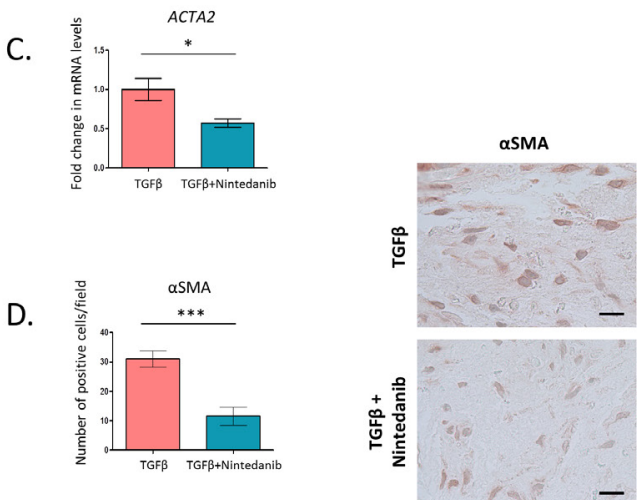

Figure 4 Inhibition of TGF $\beta$ signalling by nintedanib treatment in vascularised skin equivalent models. (A) Decreased activation of TGF $\beta$-induced SMAD signalling on nintedanib treatment as shown by immunofluorescence staining for pSMAD3 (green) and costaining with prolyl-4-hydroxylase- $\beta$ (red) and DAPI (blue), at 600-fold magnification (scale bars=25 $\mu \mathrm{m}$ ). Quantification of pSMAD3 signal intensity and HE stainings (200-fold magnification, scale bars $=100 \mu \mathrm{m}$ ) is included. (B) mRNA levels of the prototypical TGF $\beta / S M A D$ target genes PAl-1 and Smad7. (C) mRNA levels of ACTA2 (which encodes for $\alpha$-SMA). (D) Quantification of myofibroblast counts and representative $\alpha$-SMA stainings counterstained with haematoxylin at 1000 -fold magnification (scale bars $=20 \mu \mathrm{m}$ ). $\alpha$-SMA, $\alpha$-smooth muscle actin; DAPI, 4',6-diamidino-2-phenylindole; TGF $\beta$, transforming growth factor- $\beta$.

A.

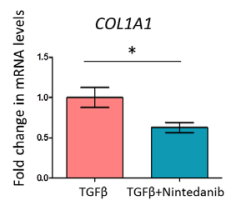

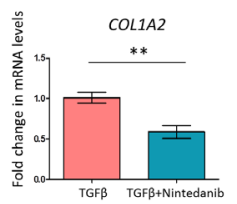

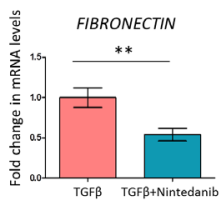

B.

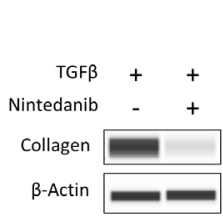

C.
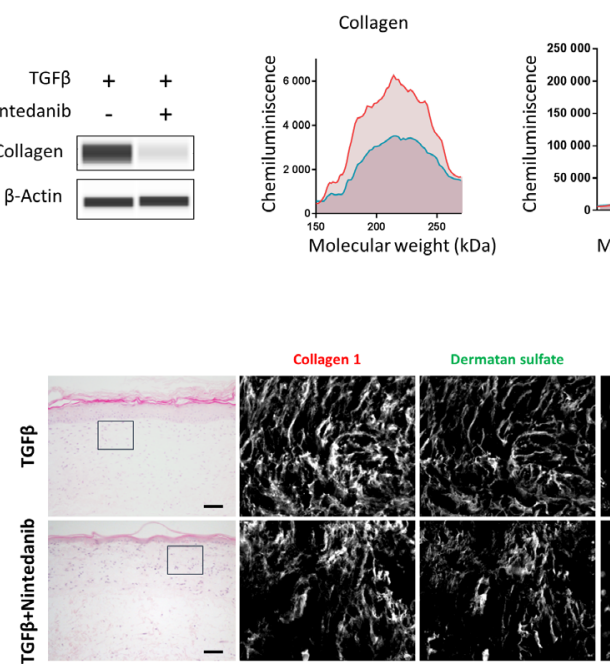
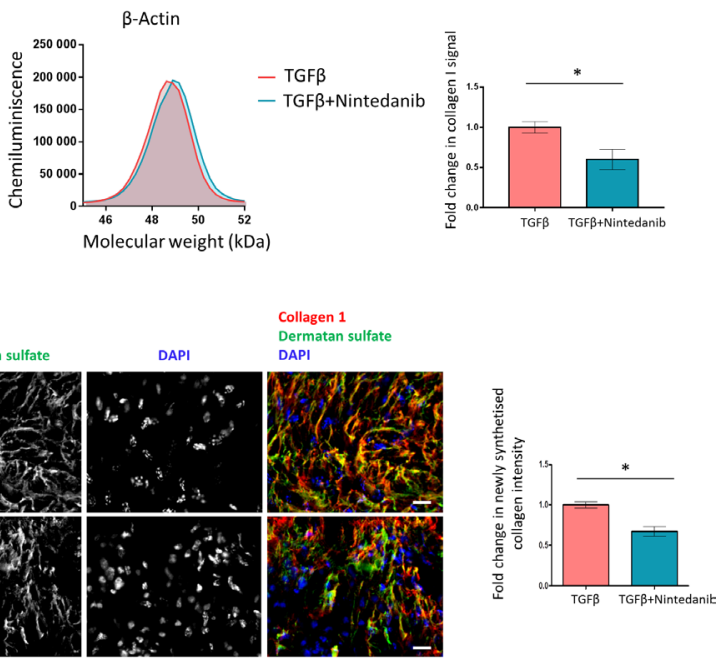

Figure 5 Antifibrotic effects of nintedanib treatment in vascularised skin equivalent models. (A) Inhibition of TGF $\beta$-induced upregulation of COL1A1, COL1A2 and fibronectin mRNA levels by nintedanib treatment. (B) Representative western blot for type I collagen, with $\beta$-actin as housekeeping gene. Chemiluminescence versus molecular weight plots and quantification are included. (C) Immunofluorescence stainings of dermatan sulfate to identify human type I collagen in the vascularised human skin equivalent, at 1000 -fold magnification (scale bars=20 $\mu \mathrm{m}$ ), counterstained with DAPI. HE stainings (200-fold magnification, scale bars $=100 \mu \mathrm{m}$ ) and quantification of collagen type I signal colocalising with dermatan sulfate are included. DAPI, 4',6-diamidino-2-phenylindole; TGF $\beta$,transforming growth factor- $\beta$. 
and enables studies of interactions between fibroblasts, endothelial cells and keratinocytes. The interplay between these cell types is a critical feature of fibrotic skin diseases like SSc. Keratinocytes are a major source of proinflammatory and profibrotic cytokines in SSc, such as interleukin (IL)-1 $1 \alpha$, IL-6, connective tissue growth factor (CTGF), oncostatin $\mathrm{M}$ and vascular endothelial growth factor (VEGF), and are involved in bidirectional double paracrine signalling with fibroblasts, thereby promoting fibroblast activation. ${ }^{18-21}$ Endothelial cells are thought to be the primary site of injury in SSc and modulate fibroblast activation by release of factors such as endothelin 1 and nitric oxide. Moreover, endothelial cells can undergo endothelial to mesenchymal transition to acquire a myofibroblast phenotype and may thus directly contribute to matrix deposition. ${ }^{22}$ Such interactions can be reproduced in vascularised skin equivalents.

We demonstrate that exposure to TGF $\beta$ induces fibrotic transformation of vascularised skin equivalents. Stimulation with TGF $\beta$ induces activation of classical profibrotic downstream mediators such as SMAD signalling and transcription of prototypical target genes. This promotes fibroblast to myofibroblast transition and ECM deposition in vascularised skin equivalents, key features of fibrotic skin in SSc. ${ }^{23}{ }^{24}$ Fibroblasts in vascularised skin equivalents can therefore acquire an activated phenotype that reproduces their aberrant activation in SSc skin.

Of particular interest, we provide first evidence that vascularised skin equivalents can serve as a platform for the evaluation of antifibrotic drugs. We used nintedanib for formal proof, as nintedanib is the first targeted antifibrotic drug that awaits approval in SSc. ${ }^{15-17}$ Treatment with nintedanib in a pharmacologically relevant dose exerted antifibrotic effects in vascularised human skin equivalents: nintedanib attenuated TGF $\beta$ signalling, reduced fibroblast to myofibroblast transition and decreased ECM deposition.

A potential alternative approach to the generation of vascularised skin equivalents from mature cell types as in our current study is the use of induced pluripotent stem cells (iPSCs). iPSCs can be rapidly expanded and can subsequently differentiated with specific protocols into the individual cell types found in human skin. ${ }^{25-30}$

Although vascularised human skin equivalents may offer novel opportunities for translational studies and drug testing, the model also has important limitations: (1) The generation of vascularised skin equivalents requires specific equipment such as an incubator with adjustable perfusion pressure, bioreactors and specific material such as decellularised porcine intestinal matrices. Since adjustable perfusion pressure is an essential feature of this model, the custom incubator is indispensable; however, matrices generated by 3D bioprinters are currently evaluated as alternatives to decellularised porcine matrices and may eventually overcome the need for them. ${ }^{31}$ (2) Generation of vascularised skin equivalents is work and time intensive, which limits the potential for high-throughput drug screening. They thus serve as confirmatory rather than as screening models. (3) Our current version of the model aims to analyse the interaction of resident cells in the skin, but omits circulating cells such as leucocytes. However, peripheral blood mononuclear cells or selected leucocyte populations of interest can be added to the circulation of vascularised skin equivalents to study crosstalk between resident cells and circulating inflammatory cells. Experiments to study the efflux of circulating leucocytes into vascularised skin equivalents are currently in preparation.

Aside from vascularised skin equivalents, alternative in vitro approaches to mimic the complex interaction in human skin and model skin fibrosis are currently developed. Most of them are lacking a functional vascular system. ${ }^{32}$ Given the importance of vascular cells in the pathogenesis of SSc, this may be an important limitation. Full-thickness skin models are 3D cell cultures that have an epidermal and a dermal component and reproduce the interaction between fibroblasts and keratinocytes. Such 3D models composed exclusively of fibroblasts and keratinocytes have been successfully used to study skin pathologies including fibrosis. ${ }^{33} 34$ Modifications of this approach that include monocytes as other pathophysiologically relevant cell types have recently been described. ${ }^{35}$

In skin-on-chip models, selected skin cells are cultured in a $3 \mathrm{D}$ microsystem. These chips can theoretically reproduce physiological structures of different complexities up to multiorgan-on-chip. ${ }^{36}$ Some of these systems include perfusion of a microfluidic channel lined by endothelial cells. However, even in these more complex skin-on-chip models, a microvascular network is lacking, thereby minimising crosstalk between endothelial cells and fibroblasts, and the physiological undulation of blood perfusion pressure cannot be reproduced. ${ }^{37} 38$

Another alternative approach to in vitro generated skin models is the ex vivo culture of skin samples. In this approach, human skin samples are taken into culture directly after the biopsies have been obtained. A major advantage of this approach is that all the cellular components including immune cells and skin structures including vessels and skin appendages are preserved in their native form. ${ }^{39-41}$ However, the biopsies can only be kept for short period in culture, before cells lose their phenotype and undergo apoptosis. Moreover, experimental manipulation such as knockdown is challenging in this setting. In addition, standardised access to human skin samples is limited, often disabling systematic larger scale studies.

We describe herein a novel human in vitro model of skin fibrosis. Decisive features of these vascularised skin equivalents include a functional vascular perfusion system; interaction with a physiological matrix; crosstalk between the major resident cells of human skin, such as fibroblasts, keratinocytes and endothelial cells; and the option to further expand cellular interactions by the addition of leucocytes. Vascularised skin equivalents thus resemble many of the key features of fibrotic skin and may not only provide novel pathophysiological insights but also serve as an advanced platform for drug testing.

\section{Author affiliations}

${ }^{1}$ Department of Internal Medicine 3 - Rheumatology and Immunology, FriedrichAlexander-University Erlangen-Nürnberg (FAU) and University Hospital Erlangen,

Erlangen, Germany

${ }^{2}$ Translational Center Würzburg, Fraunhofer Translational Center Regenerative Therapies, Fraunhofer Institute for Silicate Research (ISC), Würzburg, Germany ${ }^{3}$ Radboud Institute for Molecular Life Sciences, Department of Biochemistry, Radboud University Medical Center, Nijmegen, The Netherlands

${ }^{4}$ University for Applied Sciences Würzburg-Schweinfurt, Wurzburg, Germany

${ }^{5}$ Center of Experimental Rheumatology, University Hospital Zurich/Zurich Center of Integrative Human Physiology (ZIHP), Zurich, Switzerland

${ }^{6}$ Department for Tissue Engineering and Regenerative Medicine, Würzburg University Medical Center, Würzburg, Germany

Contributors A-EM, C-WC, FG-B and JHWD designed the study. A-EM, C-WC, LK, A-HG, Y-NL, TT-M., XX, CTM were involved in acquisition of data. A-EM, C-WC, A-HG, LK, $A J, G S, F G-B$ and JHWD were involved in interpretation of data. A-EM and JHWD were involved in manuscript preparation. THVK, JH and FG-B provided essential materials.

Funding Grants DI 1537/9-1 and DI 1537/9-2, DI 1537/11-1, DI 1537/12-1, DI 1537/13-1, DI 1537/14-1 and RA 2506/3-1 of the German Research Foundation, SFB CRC1181 (project C01) and SFB TR221/ project number 324392634 (B04) of the German Research Foundation; grants J40 and A64 of the IZKF in Erlangen; grants 2014_A47 and 2014_A184 of the Else-Kröner-Fresenius-Foundation; grant 14-12-17-1-Bergmann of the ELAN-Foundation Erlangen and a Career Support Award of Medicine of the Ernst Jung Foundation. $A J$ is a member of the SKINTEGRITY consortium at University Medicine Zürich/Hochschulmedizin Zürich. 
Competing interests JHWD has consultancy relationships and/or has received research funding from Actelion, BMS, Celgene, Bayer Pharma, Boehringer Ingelheim, JB Therapeutics, Sanofi-Aventis, Novartis, UCB, GSK, Array Biopharma and Active Biotech in the area of potential treatments of SSC and is stock owner of 4D Science $\mathrm{GmbH}$.

Patient consent for publication Not required.

Provenance and peer review Not commissioned; externally peer reviewed.

Data availability statement Data are available on reasonable request.

ORCID iDs

Alexandru-Emil Matei http://orcid.org/0000-0003-1248-3145

Georg Schett http://orcid.org/0000-0001-8740-9615

Jörg H W Distler http://orcid.org/0000-0001-7408-9333

\section{REFERENCES}

1 Distler JH, Györfi AH, Ramanujam M, et al. Shared and distinct mechanisms of fibrosis. nature reviews rheumatology in press.

2 Garrett SM, Baker Frost D, Feghali-Bostwick C. The mighty fibroblast and its utility in scleroderma research. J Scleroderma Relat Disord 2017;2:100-7.

3 Hinz B, Gabbiani G. Cell-Matrix and cell-cell contacts of myofibroblasts: role in connective tissue remodeling. Thromb Haemost 2003;90:993-1002.

4 Pakshir P, Hinz B. The big five in fibrosis: macrophages, myofibroblasts, matrix, mechanics, and miscommunication. Matrix Biol 2018;68-69:81-93.

5 Grinnell F. Fibroblast biology in three-dimensional collagen matrices. Trends Cell Biol 2003;13:264-9.

6 Rhee S. Fibroblasts in three dimensional matrices: cell migration and matrix remodeling. Exp Mol Med 2009;41:858-65.

7 Randall MJ, Jüngel A, Rimann M, et al. Advances in the Biofabrication of 3D Skin in vitro: Healthy and Pathological Models. Front Bioeng Biotechnol 2018;6.

8 Mestas J, Hughes CCW. Of mice and not men: differences between mouse and human immunology. J Immunol 2004;172:2731-8.

9 Sutoh Y, Mohamed RH, Kasahara M. Origin and evolution of dendritic epidermal T cells. Front Immunol 2018;9:1059.

10 Yue F, Cheng Y, Breschi A, et al. A comparative encyclopedia of DNA elements in the mouse genome. Nature 2014;515:355-64.

11 Groeber F, Engelhardt L, Lange J, et al. A first vascularized skin equivalent as an alternative to animal experimentation. ALTEX 2016;33:415-22.

12 Györfi AH, Matei A-E, Distler JHW. Targeting TGF- $\beta$ signaling for the treatment of fibrosis. Matrix Biology 2018;68-69:8-27.

13 Chakraborty D, Šumová B, Mallano T, et al. Activation of STAT3 integrates common profibrotic pathways to promote fibroblast activation and tissue fibrosis. Nat Commun 2017:8:1130

14 Oostendorp C, Uijtdewilligen PJE, Versteeg EM, et al. Visualisation of newly synthesised collagen in vitro and in vivo. Sci Rep 2016;6:18780.

15 Huang J, Beyer C, Palumbo-Zerr K, et al. Nintedanib inhibits fibroblast activation and ameliorates fibrosis in preclinical models of systemic sclerosis. Ann Rheum Dis 2016:75:883-90.

16 Huang J, Maier C, Zhang Y, et al. Nintedanib inhibits macrophage activation and ameliorates vascular and fibrotic manifestations in the Fra2 mouse model of systemic sclerosis. Ann Rheum Dis 2017;76:1941-8.

17 Distler O, Highland KB, Gahlemann M, et al. Nintedanib for systemic sclerosisassociated interstitial lung disease. N Eng/ J Med 2019;380:2518-28.

18 Distler O, Distler Jörg HW, Scheid A, et al. Uncontrolled expression of vascular endothelial growth factor and its receptors leads to insufficient skin angiogenesis in patients with systemic sclerosis. Circ Res 2004;95:109-16.

19 Aden N, Nuttall A, Shiwen X, et al. Epithelial cells promote fibroblast activation via IL-1alpha in systemic sclerosis. J Invest Dermatol 2010;130:2191-200.
20 McCoy SS, Reed TJ, Berthier CC, et al. Scleroderma keratinocytes promote fibroblast activation independent of transforming growth factor beta. Rheumatology 2017;56:1970-81.

21 Canady J, Arndt S, Karrer S, et al. Increased KGF expression promotes fibroblast activation in a double paracrine manner resulting in cutaneous fibrosis. J Invest Dermatol 2013;133:647-57.

22 Manetti M, Romano E, Rosa I, et al. Endothelial-To-Mesenchymal transition contributes to endothelial dysfunction and dermal fibrosis in systemic sclerosis. Ann Rheum Dis 2017;76:924-34

23 Distler JHW, Feghali-Bostwick C, Soare A, et al. Review: frontiers of antifibrotic therapy in systemic sclerosis. Arthritis Rheumatol 2017;69:257-67.

24 Beyer C, Distler O, Distler JHW. Innovative antifibrotic therapies in systemic sclerosis. Curr Opin Rheumatol 2012;24:274-80.

25 Itoh M, Umegaki-Arao N, Guo Z, et al. Generation of 3D skin equivalents fully reconstituted from human induced pluripotent stem cells (iPSCs). PLoS One 2013;8:e77673.

26 Kim Y, Park N, Rim YA, et al. Establishment of a complex skin structure via layered coculture of keratinocytes and fibroblasts derived from induced pluripotent stem cells. Stem Cell Res Ther 2018;9.

27 Wang Z, Nakamura K, Jinnin M, et al. Establishment and gene expression analysis of disease-derived induced pluripotent stem cells of scleroderma. J Dermato/ Sci 2016;84:186-96

28 Kim J-W, Kim Y, Kim J, et al. AB0189 3d skin organoid mimicking systemic sclerosis generated by patient-derived induced pluripotent stem cells: 'disease in a dish' and development of animal model. Ann Rheum Dis 2018;77:1281.2-1.

29 Takagi R, Ishimaru J, Sugawara A, et al. Bioengineering a 3D integumentary organ system from iPS cells using an in vivo transplantation model. Sci Adv 2016;2:e1500887.

30 Wimmer RA, Leopoldi A, Aichinger M, et al. Human blood vessel organoids as a model of diabetic vasculopathy. Nature 2019;565:505-10.

$31 \mathrm{Ng} \mathrm{WL}$, Wang S, Yeong WY, et al. Skin Bioprinting: impending reality or fantasy? Trends Biotechnol 2016:34:689-99.

32 van den Broek LJ, Bergers LIJC, Reijnders CMA, et al. Progress and future prospectives in Skin-on-Chip development with emphasis on the use of different cell types and technical challenges. Stem Cell Rev Rep 2017;13:418-29.

33 Wohlfahrt T, Rauber S, Uebe S, et al. Pu. 1 controls fibroblast polarization and tissue fibrosis. Nature 2019;566:344-9.

34 Rossi A, Appelt-Menzel A, Kurdyn S, et al. Generation of a three-dimensional full thickness skin equivalent and automated wounding. JoVE 2015;(96).

35 Toledo DM, Huang M, Wang Y, et al. Molecular Analysis of a Skin Equivalent Tissue Culture Model System of Systemic Sclerosis Using RNA Sequencing, Epigenetic Assays, Histology, and Immunoassays [abstract]. Arthritis Rheumatol 2018;70(suppl 10).

36 Maschmeyer I, Lorenz AK, Schimek K, et al. A four-organ-chip for interconnected long-term co-culture of human intestine, liver, skin and kidney equivalents. Lab Chip 2015;15:2688-99.

37 Abaci HE, Guo Z, Coffman A, et al. Human skin constructs with spatially controlled vasculature using primary and iPSC-derived endothelial cells. Adv Healthc Mater 2016:5:1800-7.

38 Mori N, Morimoto Y, Takeuchi S. Skin integrated with perfusable vascular channels on a CHIP. Biomaterials 2017;116:48-56.

39 Yasuoka H, Larregina AT, Yamaguchi Y, et al. Human skin culture as an ex vivo mode for assessing the fibrotic effects of insulin-like growth factor binding proteins. Open Rheumatol J 2008:2:17-22.

40 Yamaguchi Y, Takihara T, Chambers RA, et al. A peptide derived from endostatin ameliorates organ fibrosis. Sci Transl Med 2012:4:136ra71.

41 Watanabe T, Frost DB, Mlakar L, et al. A human skin model recapitulates systemic sclerosis dermal fibrosis and identifies COL22A1 as a TGF $\beta$ early response gene that mediates fibroblast to myofibroblast transition. Genes 2019;10:75. 